\title{
ON THE EXISTENCE OF WEAKLY $n$-DIMENSIONAL SPACES
}

\author{
JAN VAN MILL AND ROMAN POL
}

(Communicated by Dennis Burke)

\begin{abstract}
Using a certain method for constructing peculiar large-dimensional spaces in every compactum with sufficiently large dimension, we present for every $n$ an easy example of a weakly $n$-dimensional space.
\end{abstract}

\section{INTRODUCTION}

For a space $X$ and a point $x \in X$, ind $X$ denotes the dimension of $X$ at the point $x$ (cf. [1, Problem 1.1.B]). If $X$ is $n$-dimensional then its dimensional kernel is the set $\left\{x \in X: \operatorname{ind}_{x} X=n\right\}$. It is known that the dimensional kernel of an $n$-dimensional space $X$, where $n \geq 1$, is an $F_{\sigma}$ subset of $X$ of dimension at least $n-1$. Also, if in addition $X$ is compact, then its dimensional kernel is $n$-dimensional. For more information see [1, Problem 1.5.C]. A space $X$ is called weakly $n$-dimensional, where $n \geq 1$, if it is $n$-dimensional, but its dimensional kernel is of dimension $n-1$. Clearly, a weakly $n$-dimensional space contains no compact subspace of dimension $n$. The first examples of weakly $n$-dimensional spaces were given by Sierpiński [15] and Mazurkiewicz [9]. A simpler construction can be found in Tomaszewski [16]; he also proved that if $X$ is weakly $n$-dimensional and if $Y$ is weakly $m$-dimensional then

$$
\operatorname{dim}(X \times Y) \leq n+m-1=\operatorname{dim} X+\operatorname{dim} Y-1 .
$$

In this note we present an application of a certain method for constructing peculiar large-dimensional subspaces in every compactum with sufficiently large dimension. The technique goes back to Mazurkiewicz [9] and Knaster [3] and has been used by several authors: see Lelek [8], Zarelua [17], Rubin, Schori, and Walsh [14], Kulesza [6], Ivanov [2], Pol [13], and Krasinkiewicz [4, 5] (These papers contain further references.) We use the technique for the construction of easy weakly $n$-dimensional spaces.

Received by the editors February 14, 1990 .

1980 Mathematics Subject Classification (1985 Revision). Primary 54D45.

Key words and phrases. Mazurkiewicz's technique, dimension, essential family, weakly $n$ dimensional.

This note was partly written during the second author's visit to Vrije Universiteit (Amsterdam). He would like to thank the Department of Mathematics of this university for its hospitality. 


\section{Preliminaries}

Our terminology in dimension theory follows Engelking [1], Nagata [12], and van Mill [11].

We denote the closed interval $[0,1]$ by $I$. A compactum is a compact metrizable space. A family $\tau=\left\{\left(A_{1}, B_{1}\right),\left(A_{2}, B_{2}\right), \ldots,\left(A_{n}, B_{n}\right)\right\}$ of pairs of disjoint closed sets in a space $X$ is called essential if for every family $\left\{L_{i}: i \leq n\right\}$, where $L_{i}$ is a partition between $A_{i}$ and $B_{i}$ for every $i$, we have $\bigcap_{i \leq n} L_{i} \neq \varnothing$; if $\tau$ is not essential then it is called inessential. Recall that $X$ is at least $n$ dimensional if and only if $X$ contains an essential family of size $n$.

We shall need the following well-known fact.

2.1. Lemma. Let $K$ be $a(k+1)$-dimensional compactum and $A, B$ a pair of disjoint closed subsets of $K$. Then there exists a continuous function $f: K \rightarrow I$ taking $A$ to 0 and $B$ to 1 such that $\operatorname{dim} f^{-1}(t) \leq k$ for each $t \in\left[\frac{1}{3}, \frac{2}{3}\right]$.

This lemma follows immediately from Hurewicz's theorem (Kuratowski [7, §45]) that the zero-dimensional maps $g: K \rightarrow I^{k+1}$ are dense in the function space $C\left(K, I^{k+1}\right.$ ) (if $p: I^{k+1} \rightarrow I$ is the projection and $g: K \rightarrow I^{k+1}$ is zerodimensional then $\operatorname{dim}\left[(p \circ g)^{-1}(t)\right] \leq k$ for all $\left.t \in I\right)$. Alternatively, one can use Nagata's metric on $K$ [12, Theorem V.4]. For the purpose of $\S 2$ it is enough to know that $\operatorname{dim} f^{-1}(t) \leq k$ for $t$ belonging to a Cantor set $C$ in $I$ and this can be proved directly by a standard Urysohn construction as the points $t$ with $\operatorname{dim} f^{-1}(t) \leq k$ form a set of type $G_{\delta}$.

We will also need the following triviality: if $X$ is an $n$-dimensional $\sigma$ compact space, where $n \geq 0$, then there exists a zero-dimensional $\sigma$-compact set $N \subseteq X$ such that $\operatorname{dim}(X \backslash N) \leq n-1$. This can be verified easily by induction on $n$ : note that $\operatorname{dim} X=\operatorname{ind} X$ and apply [11, Theorem 4.7.3].

The following lemma is probably well known: its easy proof is included for the sake of completeness.

2.2. Lemma. Let $X$ and $Y$ be compact spaces, and let $f: X \rightarrow Y$ be a continuous surjection. If $Y$ is zero-dimensional at $y \in Y$ and if $\operatorname{dim} f^{-1}(y)=0$ then $X$ is zero-dimensional at every point of $f^{-1}(y)$.

Proof. Pick an arbitrary $x \in f^{-1}(y)$ and let $U$ be a neighborhood of $x$ in $X$. Since $\operatorname{dim} f^{-1}(y)=0$ there is an open and closed subset $C$ of $f^{-1}(y)$ such that $x \in C \subseteq U$. Pick disjoint open subsets $E$ and $F$ in $X$ such that

$$
E \cap f^{-1}(y)=C \text { and } F \cap f^{-1}(y)=f^{-1}(y) \backslash C .
$$

It is clear that without loss of generality we may assume that $E \subseteq U$. Observe that $E \cup F$ is a neighborhood of $f^{-1}(y)$ in $X$. Consequently, since $f$ is a closed map, there is a neighborhood $V$ of $y$ in $Y$ such that $f^{-1}(V) \subseteq$ $E \cup F$. Since $Y$ is zero-dimensional at $y$, we may assume without loss of 
generality that $V$ is open and closed. Then $f^{-1}(V) \cap E$ is an open and closed neighborhood of $x$ in $X$ which is contained in $U$.

\section{EASY WEAKLY $n$-DIMENSIONAL SPACES}

The aim of this section is to present new examples of weakly $n$-dimensional spaces.

3.1. Theorem. Let $K$ be an $(n+1)$-dimensional compactum, where $n \geq 1$. Then $K$ contains a weakly $n$-dimensional $G_{\delta}$-subset $X$ (hence $X$ is completely metrizable).

Proof. Let $\tau=\left\{\left(A_{0}, B_{0}\right), \ldots,\left(A_{n}, B_{n}\right)\right\}$ be an essential family in $K$. By Lemma 2.1 there exists a continuous function $f: K \rightarrow I$ taking $A_{0}$ to 0 and $A_{1}$ to 1 such that there exists a Cantor set $\Delta \subseteq(0,1)$ such that $\operatorname{dim} f^{-1}(t) \leq n$ for every $t \in \Delta$.

We now closely follow a construction in Rubin, Schori, and Walsh [14]. Denote the hyperspace of $K$ by $2^{K}$ and put

$$
\mathscr{C}=\left\{C \in 2^{K}: C \text { is a continuum from } A_{0} \text { to } B_{0}\right\} \text {. }
$$

Then $\mathscr{C}$ is closed in $2^{K}$ and hence is a compact space (see [11, Claim 1 of Theorem 4.7.10]). Consequently, there is a continuous surjection $\varphi: \Delta \rightarrow \mathscr{C}$. Put

$$
Z=\bigcup\left\{f^{-1}(t) \cap \varphi(t): t \in \Delta\right\} .
$$

Then $Z$ is closed in $K$, hence is compact, and $f[Z]=\Delta$ ([11, Claim 2 of Theorem 4.7.10]). Then $\operatorname{dim} Z \geq n$ because $Z$ intersects every continuum from $A_{0}$ to $B_{0}$ ([11, Corollary 4.7.9]). In addition, $\operatorname{dim} Z \leq n$ because the fibers of the restriction $g$ of $f$ to $Z$ are at most $n$-dimensional and $\Delta$ is zero-dimensional [1, Theorem 1.12.4]. We conclude that $\operatorname{dim} Z=n$.

Now let $\Lambda$ denote the dimensional kernel of $Z$. Then because $Z$ is compact, $\Lambda$ is an $n$-dimensional $\sigma$-compact subset of $Z$, cf. the remarks in $\S 2$. There exists consequently a $\sigma$-compact zero-dimensional subset $N$ of $\Lambda$ such that $\operatorname{dim}(\Lambda \backslash N) \leq n-1$, cf. the remark preceding Lemma 2.2. Now put $X=$ $Z \backslash N$. Then $X$ is clearly a $G_{\delta}$-subset of $X$ and we claim that it is weakly n-dimensional.

Let $C \in \mathscr{C}$. We will prove that $C$ meets $X$. Pick $t \in \Delta$ such that $\varphi(t)=C$. Observe that $g^{-1}(t) \subseteq C \cap Z$. If $\operatorname{dim} g^{-1}(t)>0$ then $g^{-1}(t)$ intersects $X$ because the complement of $X$ in $Z$ is zero-dimensional. We may therefore assume that $\operatorname{dim} g^{-1}(t)=0$. But since $\Delta$ is zero-dimensional, we now obtain that $Z$ is zero-dimensional at all points of $g^{-1}(t)$ (Lemma 2.2). Consequently, $g^{-1}(t) \cap \Lambda=\varnothing$, that is, $\varnothing \neq g^{-1}(t) \subseteq X$. We conclude from [11, Corollary 4.7.9] that $\operatorname{dim} X \geq n$. However, because $X$ is a subspace of $Z$, we also have $\operatorname{dim} X \leq n$. Consequently, $\operatorname{dim} X=n$, as required. 
We next prove that $X$ is weakly $n$-dimensional. This is however a triviality. Simply observe that if $x$ is a point of $X$ at which $X$ is $n$-dimensional, then $Z$ is $n$-dimensional at $x$, which implies that $x \in X \cap \Lambda$. Since by construction, $\operatorname{dim}(X \cap \Lambda) \leq n-1$, we are done.

3.2. Remark. Observe that the spaces $X$ constructed in Theorem 3.1 are rimcompact, that is, have a basis consisting of open sets with compact boundaries. This is clear because the complement of $X$ in $K$ is zero-dimensional.

3.3. Remark. If one takes $K=I^{n+1}$ in the above theorem then there is no need to use Lemma 2.1 in the proof. It seems that this gives us the easiest known examples of weakly $n$-dimensional spaces.

3.4. Remark. It seems useful to recall that the main points of the original construction of Mazurkiewicz [10]. Let $C$ be the Cantor set and consider $K=C \times I^{n}$. Let $\mathscr{I}$ denote the subspace of $2^{K} \times 2^{K} \times \cdots$ consisting of all sequences $\left(A_{1}, A_{2}, \ldots\right)$ such that $A_{1} \subseteq A_{2} \subseteq \ldots$. Then $\mathscr{I}$ is compact, so there is a continuous surjection $\varphi: C \rightarrow \mathscr{I}$. For $t \in C$ we write $\varphi(t)=$ $\left(\varphi_{1}(t), \varphi_{2}(t), \ldots\right)$. Let $\mathscr{C}_{i}=\left\{t \in C: \varphi_{i}(t) \cap\left(\{t\} \times I^{n}\right) \neq \varnothing\right\}, D_{1}=C_{1}$, $D_{i+1}=\mathscr{C}_{i+1} \backslash \mathscr{C}_{i}$, and let

$$
M=\left\{(t, x): t \in D_{i}, x \in \varphi(t) \cap\left(\{t\} \times I^{n}\right)\right\} .
$$

Then $M \subseteq C \times I^{n}$ has the following property: for each $S \subseteq M$ which projects onto $C$, each $G_{\delta}$-set $G$ in $C \times I^{n}$ containing $S$, also contains some section $\{t\} \times I^{n}$. This can be seen as follows. If $\left(C \times I^{n}\right) \backslash G=F_{1} \cup F_{2} \cup \cdots, F_{i} \in 2^{K}$, $F_{1} \subseteq F_{2} \subseteq \cdots$, then pick $t \in C$ such that $\varphi(t)=\left(F_{1}, F_{2}, \ldots\right)$. Then $t \notin \mathscr{C}_{i}$ for all $i$, hence $\{t\} \times I^{n} \subseteq G$; in particular, $\operatorname{dim} S \geq n$.

\section{REFERENCES}

1. R. Engelking, Dimension theory, PWN, Warszawa, 1978.

2. A. V. Ivanov, An example concerning a theorem of Mazurkiewicz, Seminar on General Topology, Moskov. Gos. Univ., Moscow, 1981, pp. 49-51.

3. B. Knaster, Sur les coupures biconnexes des espaces euklidiens de dimension $n>1$ arbitraire, Mat. Sb. 19 (1946), 9-18.

4. J. Krasinkiewicz, Essential mappings onto products of manifolds, Geometric and Algebraic Topology (H. Toruńczyk, S. Jackowski, and S. Spież, eds.), Banach Center Publications, vol. 18, PWN, Warszawa, 1986, pp. 377-406.

5. __ Homotopy separators and mapping into cubes, Fund. Math. 131 (1988), 149-154.

6. J. Kulesza, The dimension of products of complete separable metric spaces, Fund. Math. (to appear).

7. K. Kuratowski, Topology II, Academic Press, New York, 1968.

8. A. Lelek, Dimension inequalities for unions and mappings of separable metric spaces, Colloq. Math. 23 (1971), 69-91.

9. S. Mazurkiewicz, Sur les problèmes $\kappa$ et $\lambda$ de Urysohn, Fund. Math. 10 (1927), 311-319.

10. __ Sur les ensembles de dimension faible, Fund. Math. 13 (1929), 210-217.

11. J. van Mill, Infinite-dimensional topology: Prerequisites and introduction, North-Holland, Amsterdam, 1989. 
12. J. I. Nagata, Modern dimension theory (revised and extended edition), Heldermann Verlag, Berlin, 1983.

13. R. Pol, Countable dimensional universal sets, Trans. Amer. Math. Soc. 297 (1986), 255-268.

14. L. Rubin, R. M. Schori, and J. J. Walsh, New dimension-theory techniques for constructing infinite-dimensional examples, General Topology Appl. 10 (1979), 93-102.

15. W. Serpinski, Sur les ensembles connexes et non connexes, Fund. Math. 2 (1921), 81-95.

16. B. Tomaszewski, On weaky n-dimensional spaces, Fund. Math. 103 (1979), 1-8.

17. A. V. Zarelua, On hereditarily infinite-dimensional spaces, Theory of Sets and Topology, memorial volume in honor of Felix Hausdorff, Berlin, 1972, pp. 509-525. (Russian)

Faculteit Wiskunde en Informatica, VRije Universiteit, De BoelelaAn 1081, $1081 \mathrm{HV}$ AMSTERDAM, THE NeTHERLANDS

Wydzial Nat, U.W., PKiN 1X P., 00-901 Warszawa, Poland 